\title{
Four Weeks of Muscular Endurance Resistance Training Does Not Alter Fatigue Index
}

John W. Farrell, Daniel J. Blackwood, Rebecca D. Larson,

Department of Health and Exercise Science, University of Oklahoma 1401 Asp Ave, Norman, OK 73019, USA

Corresponding Author: John Farrell, E-mail: John.W.Farrell-1@ou.edu

\section{ARTICLE INFO}

\section{Article history}

Received: May 02, 2018

Accepted: June 25, 2018

Published: July 31, 2018

Volume: 6 Issue: 3

Conflicts of interest: None

Funding: None

\begin{abstract}
Background of Study: The implementation of a muscular endurance resistance training (MERT) program has been shown to be beneficial in augmenting the onset of blood lactate accumulation (OBLA). However, the effects of MERT on local muscular endurance has not been investigated. Objectives: The purposes of the current study were to investigate the effects of 4 weeks of MERT on local muscular endurance, and the relationship between OBLA and fatigue index (FI). Methods: Endurance trained males were randomly designated to either the experimental (EX) or control $(\mathrm{CON})$ group: $9 \mathrm{EX}$ and $8 \mathrm{CON}$. All participants continued current aerobic training. Baseline measures included OBLA, 1 repetition maximum (1RM) for: leg press (LP), leg curl (LC), and leg extension (LE). FI of the quadriceps was assessed via a dynamometer and the Thorstesson protocol. In addition, the EX group performed supervised MERT training for four weeks. A two way ANOVA was used to assess group and time differences in performance measures. The relationship between OBLA and FI was assessed using Pearson's Correlation. Results: No significant group differences were observed in all baseline measurements $(\mathrm{p}>0.05)$. There were no significant group or time differences for OBLA and FI ( $>00.05)$. Pearson's correlation revealed no significant relationship $(\mathrm{p}>0.05, \mathrm{r}=<0.01)$ existed between FI and OBLA. Conclusions: It was observed that four weeks of MERT provided an insufficient stimulus to improve OBLA and FI, and no significant relationship existed between OBLA and FI. Athletes using MERT should include additional mesocycles for favorable adaptations to local muscular endurance and OBLA.
\end{abstract}

Key words: Lactate, Physical Endurance, Strength Training, Fatigue

\section{INTRODUCTION}

Endurance sports and events consist of performing muscular contractions at submaximal intensities for either a prolonged period of time or for numerous repetitions. The nature and physiological demands of endurance sports enhance the susceptibility of participants to the development of fatigue. Due to the complex nature of fatigue several different definitions are used to describe it in the field of exercise physiology. One that seems to encompass its many facets defines fatigue as "the sensation of tiredness and associated decrements in muscular performance and function (Abbiss \& Laursen, 2005; Green, 1997; Kay et al., 2001).” Performance in most endurance sports or events is mainly determined by the maximal sustainable power or speed/pace for a given competition distance (Rønnestad \& Mujika, 2014). As fatigue develops, the ability to maintain greater power production or speed/pace is inhibited (Abbiss \& Laursen, 2005; Rønnestad $\&$ Mujika, 2014). Due to this inhibition, endurance athletes and coaches focus on utilizing training methodologies that enhance performance through improved fatigue resistance. Previous research suggests metabolite, e.g. lactate, accumu- lation during exercise exacerbates the development of fatigue. A strong correlation has been shown to exist between increases in lactate concentration and decreases in cycling performance (Abbiss \& Laursen, 2005; Ainsworth, Serfass, \& Leon, 1993; Liedl, Swain, \& Branch, 1999). An important threshold used by researchers and practitioners to mark shifts and improvements in lactate kinetics is the onset of blood lactate accumulation (OBLA) (Santos-Concejero et al., 2013; TANAKA, 1990). OBLA is the exercise intensity that corresponds to $4 \mathrm{mmol} / \mathrm{L}$ of blood lactate concentration, and also marks the transition from a tolerable exercise intensity or workload to one that is considered severe (Chmura \& Nazar, 2010; Figueira, Caputo, Pelarigo, \& Denadai, 2008). A rightward shift in OBLA to a higher workload or percentage of $\mathrm{VO}_{2} \max$ will theoretically result in reduced levels of fatigue and improved endurance performance (Faude, Kindermann, \& Meyer, 2009; Hostrup \& Bangsbo, 2017).

Evidence shows that endurance training supplemented with resistance training has an additive effect on endurance performance through enhanced improvements in exercise economy, anaerobic capacity, maximal power and speed/pace (Farrell III, Lantis, Ade, Bemben, \& Larson, 2017; Farrell III, Lantis, Ade, 
Cantrell, \& Larson, 2018; Lantis, Farrell, Cantrell, \& Larson, 2017; Losnegard et al., 2011; Rønnestad \& Mujika, 2014). Resistance training utilizing higher repetitions with lower resistance and short recovery periods between sets has been used as a potential stimulus for enhancing muscular endurance and fatigue resistance (Farrell III et al., 2017; Farrell III et al., 2018; Lantis et al., 2017). Muscular endurance resistance training (MERT) consists of performing multiple sets of 12 to 15 repetitions at $50 \%$ of $1 \mathrm{RM}$ with $\leq 1$ minute of rest between sets of exercise (Farrell III et al., 2017; Farrell III et al., 2018; Lantis et al., 2017). A bout of MERT has been shown to induce an accumulation of blood lactate to values similar to that reported by HIT, and induce improvements in OBLA and 1RM strength when combined with aerobic training in aerobically active individuals (Edge, Hill-Haas, Goodman, \& Bishop, 2006; Farrell III et al., 2017; Farrell III et al., 2018; Lantis et al., 2017; Rogatzki, Wright, Mikat, \& Brice, 2014). However, there has been little research into the effects a concurrent training program, consisting of both endurance and MERT, has on local muscular endurance and how muscular endurance relates to endurance performance variables. It can be speculated that a positive relationship between muscular endurance and OBLA exists. Individuals that are capable of maintaining high levels of strength over several repetitions theoretically should possess enhanced lactate kinetics. Also, it can be speculated that performing MERT, which has been shown to improve OBLA, should increase fatigue resistance as indicated by sustained strength while performing several repetitions. Therefore, the purpose of the current study is to examine the relationship between OBLA and local muscular endurance. Additionally, the current study aims to investigate the adaptations of both local muscular endurance and OBLA to a mesocycle (4 weeks) of MERT. The current researchers hypothesize that local muscular endurance and OBLA will exhibit a significant relationship. Also, it is hypothesized that both local muscular endurance and OBLA will exhibit significant improvements following one mesocycle of MERT.

\section{METHODS}

\section{Subjects and Design of Study}

Nineteen participants (18-37 years old) were recruited for this randomized controlled trial. 17 participants completed the study with 2 participants dropping out due to time commitment issues. Subjects were randomly divided into an experimental (EX) group, whose regular aerobic training was supplemented with a MERT program, or a control (CON) group, who continued their regular aerobic exercise training without the addition of MERT. All subjects were considered to be aerobically active and had not participated in any resistance training for 6 months prior to start of the study, determined by a self-reported physical activity questionnaire. Aerobically active was defined as having participated in aerobic exercise at least one hour per day for 3 days per week for the past 6 months. The current cohort consisted of 8 runners, 8 cyclist, and 1 triathlete. All subjects were required to reach a $\mathrm{VO}_{2} \max$ of at least $40 \mathrm{ml} / \mathrm{kg}$ / min to participate in the study. This threshold has been used previously in similar studies and indicates an aerobic capacity of good/excellent (Farrell III et al., 2017; Farrell III et al., 2018;
Heyward, 1992; Lantis et al., 2017). This study was approved by the Institutional Review Board at the University of Oklahoma; each subject gave a verbal and written informed consent before participation. All testing and training was completed in an air-conditioned laboratory at a temperature $20-25^{\circ} \mathrm{C}$.

\section{Experimental Protocol}

Prior to and following the 4 week training period both groups performed a staged graded exercise test to determine OBLA and $\mathrm{VO}_{2}$ max. The Thorstennson protocol was used to assess the fatigue rate of the knee extensor muscle group of the dominant leg (Thorstensson \& Karlsson, 1976). All subjects were instructed to continue their low-intensity aerobic training without making alterations to volume or intensity. In addition to their current low-intensity aerobic training the EX group returned to the laboratory two times per week for four weeks to perform MERT under the supervision of the researchers.

\section{Incremental exercise test}

The incremental exercise test protocol utilized has been described elsewhere (Farrell III et al., 2017; Farrell III et al., 2018; Lantis et al., 2017). In short, a resting fingertip capillary blood sample was collected to determine whole blood lactate concentration prior to testing using a commercial lactate meter (Lactate Plus, Nova Biomedical, Waltham, MA). The staged exercise test was initiated at a work rate of 125 watts (W) and increased by $25 \mathrm{~W}$ every three minutes until the participant reached task failure indicated by a pedal rate dropping below 50 revolutions per minute. At the end of each of the three-minute stages blood lactate and rating of perceived exertion (RPE), based on the Borg Scale, were measured (Borg, 1971). The $\mathrm{W}$ corresponding to $4.0 \mathrm{mmol} / \mathrm{L}$ (OBLA) was calculated by plotting $\mathrm{W}$ against blood lactate concentration and using linear interpolation (Faude et al., 2009). Metabolic and ventilatory data were continuously measured and averaged over 30 second intervals.

\section{Fatigue index}

The fatigability of the knee extensors were assessed following the Thorstensson protocol using a Kin Com dynamometer (KinCom model: KC125AP, Isokinetic International, East Ridge, TN 37412) (Thorstensson \& Karlsson, 1976). Subjects performed 50 knee extensions with the dominant leg from the knee joint angle of $90^{\circ}$ to $0^{\circ}$ (knee fully extended). Constant angular velocity was set at $180^{\circ} \mathrm{s}$. Subjects were instructed to perform extensions with maximal effort and to return to the starting position passively between contractions. Peak force from the load range portion of each contraction was recorded at $1000 \mathrm{~Hz}$. Fatigue Index (FI) was calculated using the following equation:

Fatigue Index $=\left[\begin{array}{l}(\text { Average of initial three extensions })- \\ \text { (Average of final three extensions })\end{array}\right] \times 100$




\section{Aerobic exercise}

Based on previous studies, subjects were instructed to maintain current aerobic training volume and intensity throughout the study (Farrell III et al., 2017; Farrell III et al., 2018; Karsten, Stevens, Colpus, Larumbe-Zabala, \& Naclerio, 2016; Lantis et al., 2017). If subjects indicated a decrease of $>1 / 3$ or an increase of $>5 \%$ in training volume they were excluded from the study to prevent detraining or additional aerobic adaptations (Fleck, 1994; Haff \& Triplett, 2015; Zupan \& Petosa, 1995). Also, any indications of an increase in aerobic training intensity resulted in exclusion from the study.

\section{Muscular endurance resistance training}

The current training program has been described elsewhere (Farrell III et al., 2017). In short, the training program consisted of supervised sessions on 2 days* $\mathrm{wk}^{-1}$ over a four week period to supplement participants' aerobic training program. Participants were instructed to not perform any resistance training outside of the study. Each resistance training session included a warmup consisting of several repetitions at 30 to 40 percent of their 1RM followed by four sets of 12 to 15 repetitions at $50 \%$ of the subject's established 1-RM for LP, LC, and LE (Cybex Strength Systems) with 30 seconds of rest between sets and 3 minutes of rest between exercises. If an individual was able to complete four sets of 15 repetitions after 2 weeks (4 training sessions) the training weight was increased by 4.54 kilograms (a 2 to $4 \%$ increase) to compensate for any strength gains (Haff \& Triplett, 2015; Kraemer \& Ratamess, 2004). If participants were unable to complete four sets of 15 repetitions after two weeks the training weight was kept the same.

\section{Statistical analysis}

Data are presented as means $\pm \mathrm{SD}$. Independent t-tests were utilized to determine if significant between group differences existed for subject characteristics. Data was found to be normally distributed. To determine differences in physiological variables between groups at pre- and post-training a twoway repeated measures analysis of variance (ANOVA) (time and group as factors) was utilized. If the ANOVA reached significance, a Bonferroni-post hoc analysis was performed. Pearson's r correlations were performed to determine the relationship between FI and performance measurements assessed during the GXTs. All statistical analyses were performed using SigmaPlot (Version 12.5, Systat Software Inc., San Jose, CA). Cohen's d effect sizes (ES) were reported for all significant measures. A value of $\leq 0.20$ was considered a weak effect, a value of $\leq 0.50$ was considered a moderate effect, and a value of $\geq 0.80$ was considered a strong effect (Cohen, 1988). An alpha level of $\mathrm{p} \leq 0.05$ was set for the level of significance.

\section{RESULTS}

\section{Subject Characteristics and Training Compliance}

Descriptive characteristics are summarized in Table 1. No statistical differences $(p>0.05)$ existed between groups for age, height, and body mass. Of the 9 subjects included in the EX group all participants completed at least 12 repetitions per set for all training sessions. All subjects met the requirements for increasing training load after the $4^{\text {th }}$ training session.

\section{Performance Measurements}

Performance measures collected and assessed are presented in Table 2. No statistically significant differences $(\mathrm{p}>0.05)$ were present between groups for $\mathrm{VO}_{2} \max , \mathrm{OBLA}$, and $\mathrm{FI}$ prior to the training intervention. Post-training statistical analysis indicated no significant group $\mathrm{X}$ time interaction for all performance variables with no main effects for group or time.

\section{Correlations}

Pearson's r correlations were performed to examine the relationship between FI and OBLA. Since no significant differences were observed between groups and between pre- and post-training, both groups and time points were pooled for analysis. It was revealed that no statistically significant relationship between FI and OBLA $(r=-0.01$ and $p=0.44)$ exists. This relationship is illustrated in Figure 1.

\section{DISCUSSION}

The purposes of the current study were: (1) to examine the effects of aerobic exercise supplemented with 4 weeks of MERT on local muscular endurance, assessed via the FI of the quadriceps, and (2) to examine the relationship between local muscular endurance and variables related to endurance performance, OBLA and $\mathrm{VO}_{2}$ max. Analysis from the current study revealed no significant improvements in FI in following the prescribed training intervention. Additionally, no significant relationships were detected between FI and the aerobic performance variables: OBLA and $\mathrm{VO}_{2}$ max. The current study did not observe any significant improvements in the FI of the quadriceps following 4 weeks of MERT. This is an interesting finding as previous research pertaining to resistance training using lower loads and higher repetitions have seen improvements in local muscular endurance as well as hypertrophy of Type I muscle fibers (Campos et al., 2002; Mitchell et al., 2012; Brad J. Schoenfeld, Grgic, Ogborn, \& Krieger, 2017; Brad J Schoenfeld, Peterson, Ogborn, Contreras, \& Sonmez, 2015; Vinogradova et al., 2013). Schoenfeld et al. 2015 prescribed participants to perform 3 sets of 25-35 repetitions at 30 to $50 \%$ of 1RM 3 time per week for 8 weeks (Brad J Schoenfeld et al., 2015). It was observed that after completing the training intervention participants saw a significant improvement $(16.6 \%)$ in the number of repetitions performed to failure for bench press at $50 \%$ of $1 \mathrm{RM}$. Additionally, significant increases in the muscle thickness of the elbow flexors and extensors and quadriceps were observed (8.6\%, 5.2\%, and 9.3\% respectively). Differentiation between the Type II (fast twitch) and Type I (slow twitch) fibers was not described in the study by Schoenfeld et al. 2015 (Brad J. Schoenfeld et al., 2017). Previous research has 
Table 1. Subject characteristics (Mean \pm SD)

\begin{tabular}{|c|c|c|c|c|}
\hline \multicolumn{5}{|c|}{ Groups } \\
\hline \multirow[t]{2}{*}{ Variables } & \multicolumn{2}{|c|}{ Experimental $(\mathrm{n}=9)$} & \multicolumn{2}{|c|}{ Control $(n=8)$} \\
\hline & Pre & Post & Pre & Post \\
\hline Age (years) & $21.4 \pm 2.1$ & $21.6 \pm 2.1$ & $25.5 \pm 6.4$ & $25.6 \pm 6.3$ \\
\hline Height (cm) & $180.2 \pm 5.3$ & $180.2 \pm 5.3$ & $179.5 \pm 3.9$ & $179.5 \pm 3.8$ \\
\hline Body mass (kg) & $75.2 \pm 10.0$ & $75.2 \pm 8.6$ & $77.2 \pm 11.8$ & $76.6 \pm 11.4$ \\
\hline
\end{tabular}

Differences if present were denoted using $*(\mathrm{p}<0.05)$. Standard deviations represent variability

Table 2. Physiological variables (Mean \pm SD)

\begin{tabular}{|c|c|c|c|c|c|c|}
\hline \multicolumn{7}{|c|}{ Groups } \\
\hline \multirow[t]{2}{*}{ Variables } & \multicolumn{3}{|c|}{ Experimental $(n=9)$} & \multicolumn{3}{|c|}{ Control $(n=8)$} \\
\hline & Pre & Post & $\Delta \%$ & Pre & Post & $\Delta \%$ \\
\hline $\mathrm{VO}_{2} \max (\mathrm{ml} / \mathrm{kg} / \mathrm{min})$ & $50.2 \pm 7.03$ & $50.9 \pm 7.81$ & $1.22 \pm 5.81$ & $46.2 \pm 3.95$ & $47.3 \pm 4.43$ & $2.34 \pm 5.16$ \\
\hline $\operatorname{OBLA}(\mathrm{W})$ & $198.3 \pm 46.2$ & $191.1 \pm 46.5$ & $-3.69 \pm 6.08$ & $179.5 \pm 34.2$ & $183.1 \pm 37.3$ & $1.92 \pm 5.37$ \\
\hline Fatigue index (\%) & $54.0 \pm 18.3$ & $53.9 \pm 10.7$ & $-0.01 \pm 17.6$ & $57.3 \pm 13.1$ & $58.7 \pm 14.6$ & $1.40 \pm 10.8$ \\
\hline
\end{tabular}

Differences if present were denoted using $*(p<0.05)$. Standard deviations represent variability

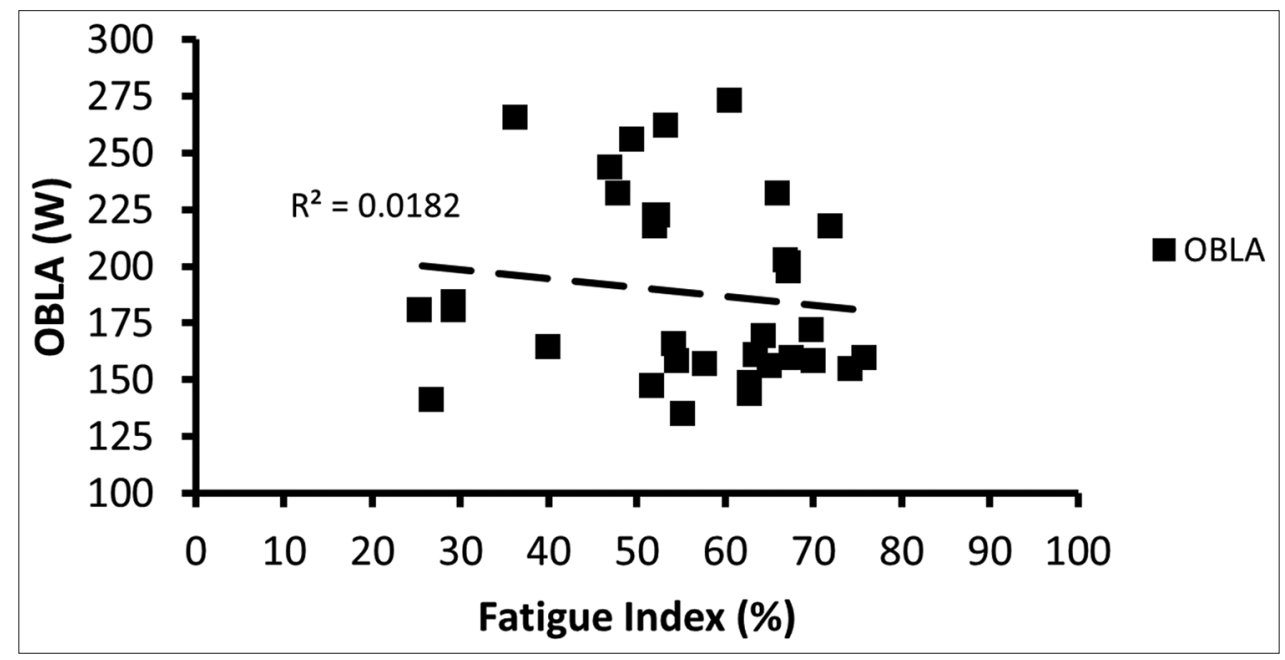

Figure 1. Relationship between FI and OBLA

demonstrated a direct relationship between the growth of muscle fiber types and training load utilized (Brad J. Schoenfeld et al., 2017; Vinogradova et al., 2013). The beneficial effects observed on muscular endurance that training with lower loads has induced in previous studies can been speculated to be partly due to favorable phenotypic adaptions, such as increases in size and proportion, to Type I muscle fibers (Brad J. Schoenfeld et al., 2017; Brad J Schoenfeld et al., 2015).

When utilizing lower loads with higher repetitions longer time-under-load occurs, resulting in a greater increase in metabolic stress compared to traditional resistance training (Burd et al., 2010; Brad J. Schoenfeld et al., 2017; Brad J Schoenfeld et al., 2015; Vinogradova et al., 2013). Metabolic stress is indicated by an increase in calcium flux as well as an accumulation in lactate and hydrogen ions. This stress has been hypothesized to mediate muscle hypertrophy. It has also been shown to favorably affect mitochondrial protein synthesis that may enhance cellular energetics in a manner that favors fatigue resistance. MERT has been shown to induce a significant increase in metabolic stress indicated by large accumulations of lactate (Farrell III et al., 2017). Theoretically, this accumulation should have provided a stimulation of the proper magnitude to induce adaptations associated with fatigue resistance. However, differences in the volume of stimulus provided through resistance training differs between the current study and previous research, and could provide an explanation for the lack of favorable adaptations (Farrell III et al., 2018; Lantis et al., 2017; Brad J Schoenfeld et al., 2015; Vinogradova et al., 2013). The current researchers speculate that the reason for the lack of improvements in muscular endurance, as seen in previous literature, may be due to differences in length of the current training intervention, total training volume, and training status of participants compared to previous research (Farrell III et al., 2018; Lantis et al., 2017; Brad J. Schoenfeld et al., 2017; Brad J Schoenfeld et al., 2015). Previous research pertaining to MERT and resistance training utilizing low loads and high repetitions, 
which have observed significant improvements in variables related to fatigue resistance, have been at least 8 weeks in length with 2-3 training sessions per week (Farrell III et al., 2018; Lantis et al., 2017; Brad J Schoenfeld et al., 2015). The current study consisted of 2 training sessions per week for 4 weeks. The length of the current study was chosen to mimic that of the typical length of a training mesocycle. It can be concluded that 4 weeks of MERT with 2 sessions per week is not a sufficient physiological stimulus to induce fatigue resistant adaptions. The current training intervention was concurrent in nature, consisting of both MERT and aerobic training. Although several previous studies have shown that concurrent training has additive effects on endurance performance, it has been shown that concurrent training attenuates adaptations associated with resistance training. It can be speculated that the aerobic training performed in the current study may have attenuated some adaptations compared to if the resistance training had been performed independently (Farrell III et al., 2018; Lantis et al., 2017; Rønnestad \& Mujika, 2014). Previous research has speculated that the utilization of low loads during resistance training induces adaptations preferentially to the Type I muscle fibers (Brad J. Schoenfeld et al., 2017; Brad J Schoenfeld et al., 2015; Vinogradova et al., 2013). These previous studies have used both untrained and resistance trained individuals who perhaps possessed underdeveloped Type I muscle fibers, resulting in large adaptations (Brad J Schoenfeld et al., 2015). However, the current study utilized individuals who were aerobically trained, and it can be speculated that these individuals had a much greater degree of development of Type I muscle fibers or Type Ila fibers that were shifted more towards the aerobic end of the spectrum. The greater degree of aerobic adaptions in the current cohort may have blunted the response, and may indicate the need for a greater stimulus to induce a favorable response.

The current study did not observe any improvements in OBLA in the EXP group following the training intervention. Previous research using MERT in aerobically trained individuals has shown improvements in lactate kinetics via an increase in the power output and percentage of $\mathrm{VO}_{2} \max$ at which OBLA occurred during a GXT (Farrell III et al., 2018; Lantis et al., 2017). However, as previously mentioned the current training intervention was 4 weeks in length compared to 8 weeks used by previous research (Farrell et al., 2018; Lantis et al., 2017). This finding may provide additional insight into the proper length of training needed to induce favorable adaptions for both OBLA and local muscular endurance in aerobically trained individuals. Additionally, it can be speculated that the due to the current cohort participating in aerobic training they may have possessed a large percentage of Type I muscle fibers and thus an enhanced ability to remove and oxidize lactate from the blood stream. A greater training stimulus maybe required to induce any desired adaptions associated with enhancing lactate kinetics in the current cohort due to their physiological profile and previous training prior to the start of the current training intervention. The same reasoning for the lack of improvements in OBLA may also explain the lack of improvement in the FI of the quadriceps. As previously mentioned the current cohort may have possessed a large percentage of Type I muscle fibers and previous training was designed to further develop these fibers. This may have reduced the physiological impact that the current training intervention had on the cohort. When examining the relationship between FI and OBLA in the current data set, it appears that no significant relationship exists. The reasoning for the absence of a significant relationship could pertain to the nature of the Thorstensson test and lack of specificity in regards to the aerobic activities performed by the current cohort (Thorstensson \& Karlsson, 1976).

The current study is not without its limitations. The assessment of the fatigue index via the Thorstensson protocol may have lacked specificity to not only the aerobic activities that the current cohort participated in, but also the current training intervention. Future studies examining local muscular endurance following a training intervention of MERT may chose to use testing that better reflects the movements and number of repetitions used during the training intervention. Future studies examining the effects of MERT on local muscular endurance should also utilize an intervention closer in length to that used by previous studies (Farrell et al., 2018, Lantis et al., 2017).

\section{CONCLUSION}

The current study observed: 1 ) that 4 weeks ( 8 training sessions) of MERT did not induce favorable adaptions associated with improved muscular endurance and fatigue resistance and 2) that FI, assessed via the Thorstennson protocol, did not exhibit a significant relationship with OBLA and $\mathrm{VO}_{2} \max$. The current study provides insight into the proper programming of MERT for adaptions associated with fatigue resistance in aerobically active individuals. It appears that 4 weeks, or one mesocycle, is indeed not a sufficient stimulus to induce adaptations. Trainers and coaches should aim to integrate more than one mesocycle of MERT into an endurance athlete's program to allow for an appropriate length of time for adaptations.

\section{ACKNOWLEDGMENTS}

No external funding was used to support the current study. The authors have no relationships with any companies who will benefit from the results of the present study.

\section{REFERENCES}

Abbiss, C. R., \& Laursen, P. B. (2005). Models to explain fatigue during prolonged endurance cycling. Sports Medicine, 35(10), 865-898.

Ainsworth, B. E., Serfass, R. C., \& Leon, A. S. (1993). Effects of recovery duration and blood lactate level on power output during cycling. Canadian Journal of Applied Physiology, 18(1), 19-30.

Borg, G. (1971). The perception of physical performance. Frontiers of fitness, 280-294.

Burd, N. A., West, D. W., Staples, A. W., Atherton, P. J., Baker, J. M., Moore, D. R., Baker, S. K. (2010). Lowload high volume resistance exercise stimulates muscle protein synthesis more than high-load low volume resistance exercise in young men. PLoS One, 5(8), e12033. 
Campos, G. E., Luecke, T. J., Wendeln, H. K., Toma, K., Hagerman, F. C., Murray, T. F., Staron, R. S. (2002). Muscular adaptations in response to three different resistance-training regimens: specificity of repetition maximum training zones. European journal of applied physiology, 88(1-2), 50-60.

Chmura, J., \& Nazar, K. (2010). Parallel changes in the onset of blood lactate accumulation (OBLA) and threshold of psychomotor performance deterioration during incremental exercise after training in athletes. International Journal of Psychophysiology, 75(3), 287-290.

Cohen, J. (1988). Statistical power analysis for the behavioral sciences $\left(2^{\text {nd }}\right.$ ed.). Hillsdale, NJ: Erlbaum.

Edge, J., Hill-Haas, S., Goodman, C., \& Bishop, D. (2006). Effects of Resistance Training on $\mathrm{H}^{\wedge}+$ Regulation, Buffer Capacity, and Repeated Sprints. Medicine and Science in Sports and Exercise, 38(11), 2004.

Farrell III, J. W., Lantis, D. J., Ade, C. J., Bemben, D. A., \& Larson, R. D. (2017). Metabolic Response to Four Weeks of Muscular Endurance Resistance Training. International Journal of Kinesiology \& Sports Science, 5(4), 10.

Farrell III, J. W., Lantis, D. J., Ade, C. J., Cantrell, G. S., \& Larson, R. D. (2018). Aerobic exercise supplemented with muscular endurance training improves onset of blood lactate accumulation. The Journal of Strength \& Conditioning Research, 32(5), 1376-1382.

Faude, O., Kindermann, W., \& Meyer, T. (2009). Lactate threshold concepts. Sports Medicine, 39(6), 469-490.

Figueira, T. R., Caputo, F., Pelarigo, J. G., \& Denadai, B. S. (2008). Influence of exercise mode and maximal lactatesteady-state concentration on the validity of OBLA to predict maximal lactate-steady-state in active individuals. Journal of Science and Medicine in Sport, 11(3), 280-286.

Fleck, S. J. (1994). Detraining: Its Effects on Endurance and Strength. Strength \& Conditioning Journal, 16(1), 22-28.

Green, H. (1997). Mechanisms of muscle fatigue in intense exercise. Journal of Sports Sciences, 15(3), 247-256.

Haff, G. G., \& Triplett, N. T. (2015). Essentials of Strength Training and Conditioning $4^{\text {th }}$ Edition: Human kinetics.

Heyward, V. H. (1992). Advanced Fitness Assessment and Exercise Prescription. Medicine \& Science in Sports \& Exercise, 24(2), 278.

Hostrup, M., \& Bangsbo, J. (2017). Limitations in intense exercise performance of athletes-effect of speed endurance training on ion handling and fatigue development. The Journal of Physiology, 595(9), 2897-2913.

Karsten, B., Stevens, L., Colpus, M., Larumbe-Zabala, E., \& Naclerio, F. (2016). The effects of a sports specific maximal strength and conditioning training on critical velocity, anaerobic running distance and $5-\mathrm{km}$ race performance. International journal of sports physiology and performance, 11(1), 80-85.

Kay, D., Marino, F. E., Cannon, J., St Clair Gibson, A., Lambert, M. I., \& Noakes, T. D. (2001). Evidence for neuromuscular fatigue during high-intensity cycling in warm, humid conditions. European journal of applied physiology, 84(1), 115-121.
Kraemer, W.J., \& Ratamess, N.A. (2004) Fundamentals of resistance training: progression and exercise prescription. Medicine and science in sports and exercise, 36 (4), 674-688.

Lantis, D. J., Farrell, J. W., Cantrell, G. S., \& Larson, R. D. (2017). Eight Weeks of High Volume Resistance Training Improves Onset of Blood Lactate in Trained Individuals. The Journal of Strength \& Conditioning Research.

Liedl, M. A., Swain, D. P., \& Branch, J. D. (1999). Physiological effects of constant versus variable power during endurance cycling. Medicine and Science in Sports and Exercise, 31(10), 1472-1477.

Losnegard, T., Mikkelsen, K., Rønnestad, B., Hallén, J., Rud, B., \& Raastad, T. (2011). The effect of heavy strength training on muscle mass and physical performance in elite cross country skiers. Scandinavian journal of medicine \& science in sports, 21(3), 389-401.

Mitchell, C. J., Churchward-Venne, T. A., West, D. W., Burd, N. A., Breen, L., Baker, S. K., \& Phillips, S. M. (2012). Resistance exercise load does not determine training-mediated hypertrophic gains in young men. Journal of applied physiology, 113(1), 71-77.

Rogatzki, M. J., Wright, G. A., Mikat, R. P., \& Brice, A. G. (2014). Blood ammonium and lactate accumulation response to different training protocols using the parallel squat exercise. The Journal of Strength \& Conditioning Research, 28(4), 1113-1118.

Rønnestad, B. R., \& Mujika, I. (2014). Optimizing strength training for running and cycling endurance performance: A review. Scandinavian journal of medicine \& science in sports, 24(4), 603-612.

Santos-Concejero, J., Granados, C., Bidaurrazaga-Letona, I., Zabala-Lili, J., Irazusta, J., \& Gil, S. M. (2013). Onset of blood lactate accumulation as a predictor of performance in top athletes. Retos. Nuevas tendencias en Educación Física, Deporte y Recreación, 23, 67-69.

Schoenfeld, B. J., Grgic, J., Ogborn, D., \& Krieger, J. W. (2017). Strength and Hypertrophy Adaptations Between Low- vs. High-Load Resistance Training: A Systematic Review and Meta-analysis. The Journal of Strength \& Conditioning Research, 31(12), 3508-3523. doi: 10.1519/jsc.0000000000002200

Schoenfeld, B. J., Peterson, M. D., Ogborn, D., Contreras, B., \& Sonmez, G. T. (2015). Effects of low-vs. high-load resistance training on muscle strength and hypertrophy in well-trained men. The Journal of Strength \& Conditioning Research, 29(10), 2954-2963.

Tanaka, K. (1990). Lactate-related factors as a critical determinant of endurance. The Annals of physiological anthropology, 9(2), 191-202.

Thorstensson, A., \& Karlsson, J. (1976). Fatiguability and fibre composition of human skeletal muscle. Acta Physiologica, 98(3), 318-322.

Vinogradova, O. L., Popov, D. V., Netreba, A. I., Tsvirkun, D. V., Kurochkina, N. S., Bachinin, A. V., Orlov, O. I. (2013). Optimization of training: New developments in safe strength training. Human Physiology, 39(5), 511523. doi: 10.1134/s0362119713050162

Zupan, M. F., \& Petosa, P. S. (1995). Aerobic and Resistance Cross-Training for Peak Triathlon Performance. Strength \& Conditioning Journal, 17(5), 7-12. 